\title{
REQUIREMENTS FOR DIAGRAMING IN THE DESIGN OF MENTAL HEALTH DELIVERY SERVICES
}

\author{
A. Komashie ${ }^{1,2, \otimes}$ and P. J. Clarkson ${ }^{1}$ \\ ${ }^{1}$ University of Cambridge, United Kingdom, ${ }^{2}$ THIS.Institute, United Kingdom \\ $\triangle$ A.Komashie@eng.cam.ac.uk
}

\begin{abstract}
Delivering good quality mental health services remains a top priority in the English National Health Service (NHS). An approach to designing better delivery systems that takes into account the complexities of mental health services is highly desirable. This paper follows previous work that have sought to identify the key components of mental health delivery systems and explored the nature of the relationships between them. The paper presents the results of a qualitative thematic analysis of the requirements for diagrams as tools for describing and representing delivery systems in mental health.
\end{abstract}

Keywords: healthcare design, complex systems, co-design

\section{Introduction}

The quality improvement and patient safety challenges facing health systems worldwide, and the resulting calls for engineering systems and design approaches are well documented (Clarkson, 2018; Department of Health and Design Council, 2003; Reid et al., 2005; WHO, 2000). A systems approach to healthcare improvement is one that effectively combines perspectives on people, systems, design and risk (Clarkson et al., 2017). The most fundamental need in effectively applying a systems approach in healthcare is, arguably, the need to understand the problem and the system. The growing complexity of modern healthcare delivery services makes the need for understanding the system a non-trivial task. Fields such as systems engineering, software engineering and industrial engineering have spent several decades developing tools for managing process and systems complexity. Of the wide range of tools available for systems modelling - from simple diagrammatic modelling to computer simulations - we are particularly interested in the diagrammatic approach. This is because the expertise for sophisticated computer-based modelling in healthcare is limited. On the other hand, a diagrammatic approach may be relatively easier to use and yet make significant contributions to systems understanding and problem structuring.

Though there is growing evidence that the use of diagrams play an important role in healthcare improvement (Colligan et al., 2010; Jun et al., 2009), the most common tools have their origins in industrial engineering and software engineering (Mincarone et al., 2018). But the context for the creation, sharing and use of diagrams in healthcare may not be the same as in those industries. To our knowledge, no diagramming tool or approach exists that has been developed with a specific understanding of the nature of healthcare delivery systems. 
Consequently, the goal of our wider research into diagramming in mental health is to develop a deeper understanding of the setting and to develop a diagramming approach informed by that understanding. This present paper reports on the third stage of the wider research project. The main question addressed in the paper is "what are the key requirements for a diagramming approach in a mental health setting?" We used qualitative thematic analysis of focus group and interview data to derive a set of requirements for diagramming as a way of understanding mental health delivery services as systems. The paper suggests that these requirements may become a framework for assessing the quality and relevance of diagrammatic representations in mental health service redesign.

\section{Background}

The use of the term diagrams in this paper refers to the visual and planer representation of thoughts, concepts, processes or systems for the purpose of communication. The human use of visual communications involving diagrams has been recognised as dating back millennia (Tversky, 2011, 2015). Similarly, the use of formal drawings in engineering and science has a long history. However, a more rigorous use of diagrams in the design of business processes and complex engineering systems may be traced back to the beginning of the last century (del Aguila et al., 2014).

Significant research has since been conducted into the nature, uses and the role of diagrams in the design of systems and processes in several fields including engineering design (Purcell and Gero, 1998), industrial engineering (Graham, 2008), systems engineering (Object Management Group, 2011), software engineering (Störrle and Fish, 2013; Yusuf et al., 2007) and computer science (Blackwell, 2019). A considerable part of these efforts appears to have been devoted to the development of effective diagrammatic representational methods that facilitate a systematic approach to the design of complex systems. For instance, in software engineering, the search for an effective diagrammatic method took several decades before the development of the Unified Modelling Language (UML) in 1995 (del Aguila et al., 2014).

In recent decades, several researchers have become interested in the value of diagrams and the need to understand the ways in which they are perceived and used in the design of systems and processes. For example, Störrle and colleagues investigated regularities in the reading behaviours of expert and novice UML modellers using a variety of diagram types and eye-tracking devices (Störrle et al., 2018). The researchers identified three reading strategies - Diagram flow (eyes move along diagram edges), Text flow (eyes move from top-left to bottom-right as if reading text) and Random walk (no recognizable order). Interestingly, they also found that expert modellers move between the three strategies as they read Class Diagrams and Use Case Diagrams. In contrast, novice modellers predominantly used the random walk, especially when reading diagrams with bad layouts (Störrle et al., 2018). Clearly, there is more to understand in realising the full potential of diagrams in systems design.

In healthcare, the evidence suggests that the most common diagrammatic modelling tools are borrowed from industrial engineering, systems engineering or software engineering. A recent systematic review found that the most common diagrammatic tools used in healthcare are the Business Process Modelling and Notations (BPMN) and the Unified Modelling Language (UML) (Mincarone et al., 2018). Other researchers have also demonstrated that the type of diagram used by healthcare practitioners affects their ability to identify safety issues in care delivery processes (Colligan et al., 2010; Jun et al., 2009). There is, therefore, a growing interest in understanding the role of diagrams in the healthcare improvement process.

The current paper forms part of a developing story emerging from a wider research project in four stages. The research addressed what we should be mapping or describing and for what purpose? What does a healthcare delivery service consist of? What are the key components? How do the key components interact to impact on service user experience? With a focus on mental healthcare delivery services, the first stage identified ten key components of a delivery system (Komashie et al., 2017). The second stage focused on understanding the nature of the relationship between the ten components and addressed the question "how do service users and clinicians express these relationships between key system components" (Komashie and Clarkson, 2018). With the identification of the key system components and the understanding of the relationship between them, it is now desirable to find the most effective way of modelling and describing them. The focus of the present paper is therefore on 
what the requirements of an effective diagrammatic approach to describing a mental health service might be.

\section{Methodology}

The overall study design is shown in Figure 1 and the semantic stage (stage 3 ) which is the focus of the current paper is highlighted in the red box.

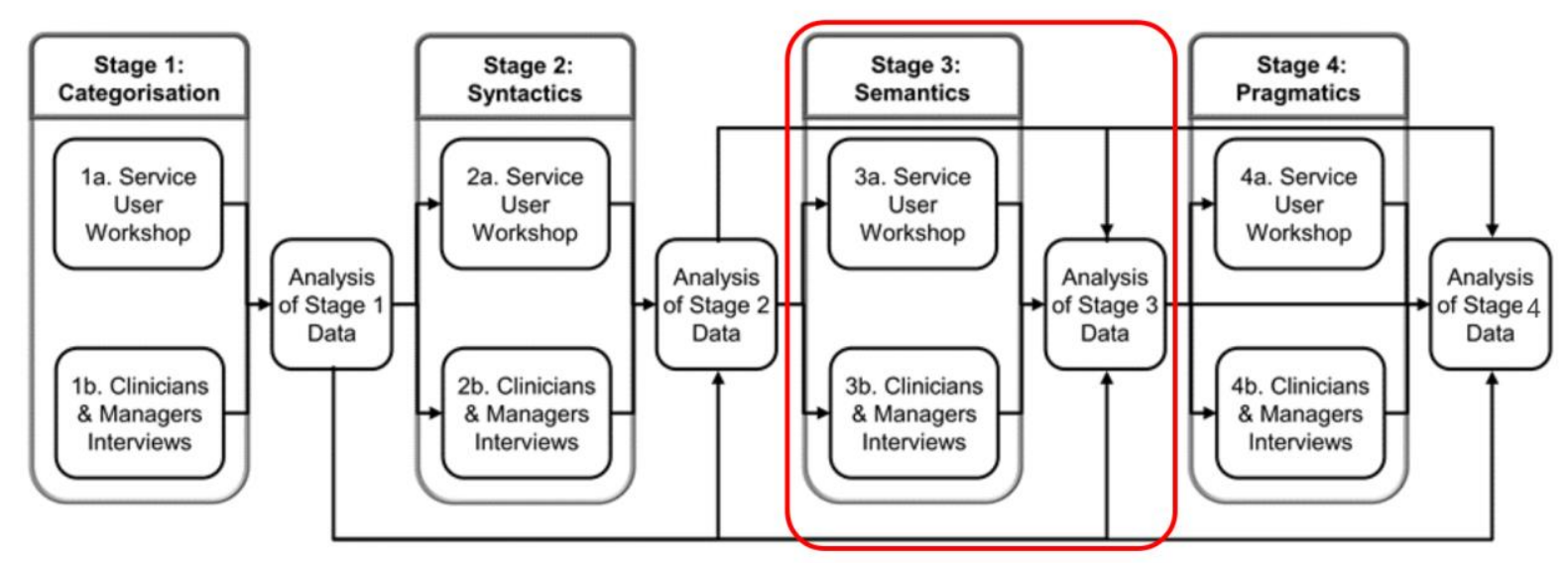

Figure 1. Research design showing semantic stage reported in this paper

The approach is broadly qualitative, and the study design adopts a semiotic framework (Ram and Liu, 2008) with the four stages of categorisation, syntactics, semantics and pragmatics. Semiotics is the science of how humans communicate with signs and was pioneered by Ferdinand de Saussure in the 1960s. The categorisation stage focused on the identification of the key components of the mental health delivery system. The syntactic stage then looked at the nature of the relationships between the system components and the semantic stage is concerned with how the components and their relationships may be represented diagrammatically. It is the requirements for such diagrammatic representation that is the focus of the current paper.

\subsection{Participants}

Participants were selected from the same NHS Mental Health Hospital in the East of England who have been involved in adult mental health services. Service user (patient) participants were recruited through research posters distributed at various clinics with telephone and email contact details. Interested service users contacted the researcher and were given further information before deciding to participate. All service user participants had capacity to consent, were reimbursed for travel and given a $£ 10$ gift voucher after each session they attended. Staff participants were recruited through an email invitation and there was no remuneration for participation. All participants had to be between the age of 16 and 65 and should have experience of receiving mental health service in the study hospital to be included. Clinicians and managers had to be currently working in the study hospital and involved with Adult Mental Health. Data was gathered through a focus group with service users $(n=6)$ and face-toface interviews with clinicians and managers $(n=5)$.

\subsection{Procedure}

The research protocol for the wider study was reviewed and approved by the Health Research Authority in England through the East of England- Cambridgeshire and Hertfordshire Research Ethics Committee (REC Ref: 16/EE/0042). In addition, the study had a strong Patient and Public Involvement (PPI) component (Staniszewska et al., 2011). We implemented PPI through the formation of a specific Service User Advisory Group (SUAG) for the project.

A researcher and one member of the SUAG co-facilitated all the focus groups with service users. The focus groups lasted between two to two and a half hours. All participants signed a consent form and 
were given information sheets. Ground rules for the session were then read and agreed. After an introduction to the study by the researcher, the SUAG member started to tell her own story of using mental health services at the study hospital, which then helped participants to come forth with their own stories or feel at ease to respond to questions.

The focus group for the stage 3 (semantics) reported in this paper had three parts - service user preferences in diagrams, service user assessment of existing diagrams and service user feedback on the experience of participation in the focus group. For the first part, the group spent some time discussing a detailed textual description of a mental health in-patient unit for young people in the study hospital. Following the discussion, service users were then presented with a flowchart (shown on the left side of Figure 2) of the processes within the same unit and encouraged to express their views on what they liked about the flowchart and what they felt was missing.

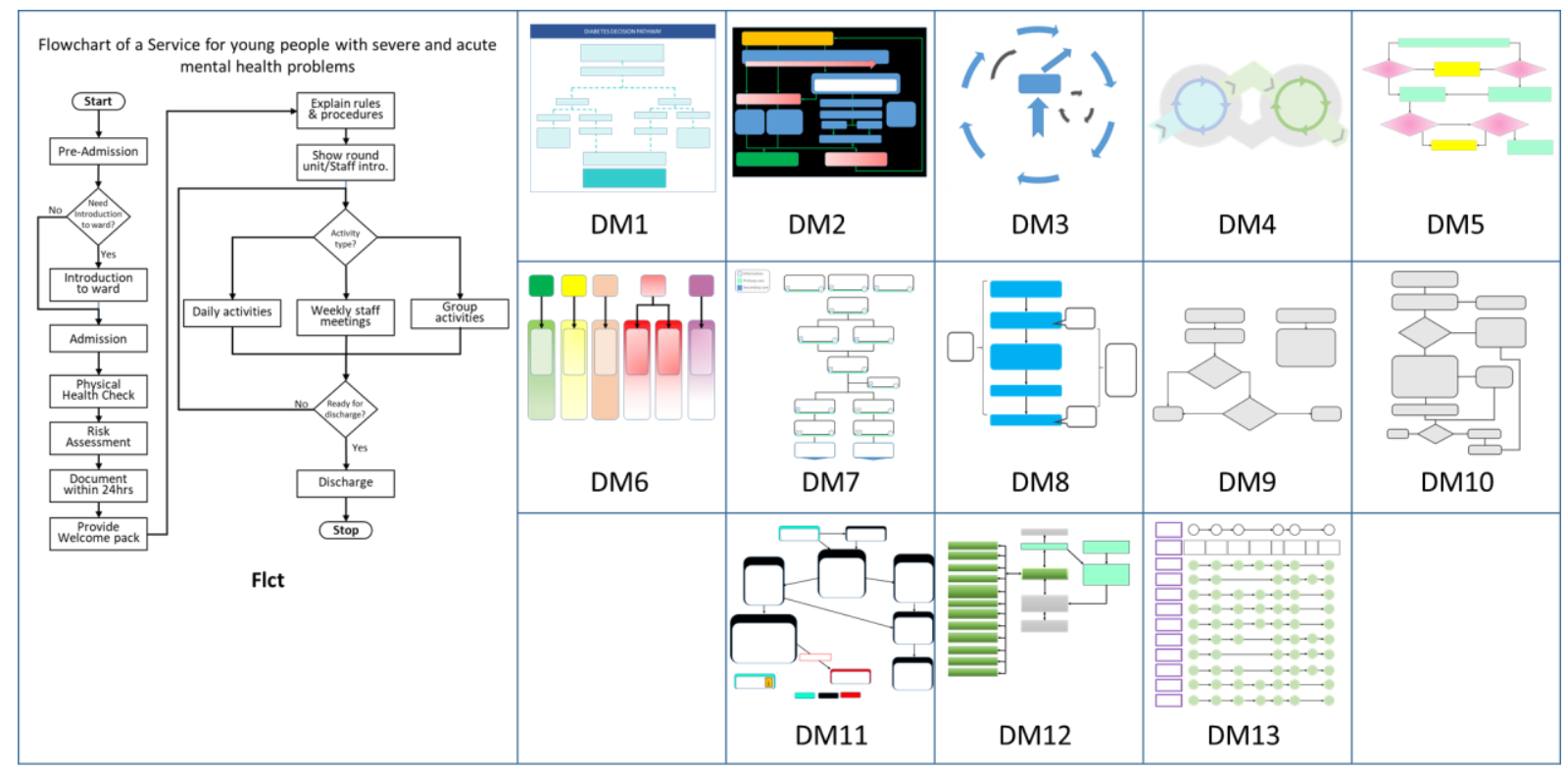

Figure 2. Variety of diagrams used in focus groups and interviews (redrawn without text from originals)

The second part focused on assessing service users' preferences concerning diagrammatic representations. This involved the use of graphic elicitation techniques (Crilly et al., 2006) by presenting the group with a random selection of diagrams from a wide range of health applications. These were selected from the internet and have been redrawn without text in Figure 2 above. Participants were encouraged to express their likes and dislikes of each of the diagrams and most importantly to say why. The service users were able to discuss and comment on diagram DM1 to DM6 in addition to the flowchart. Participants filled out a short feedback questionnaire before the session was drawn to a close. Five staff interviews were conducted for this stage in addition to the focus group. The content and format of the interviews were the same as the focus group but adapted for a face-to-face interview situation. The duration for the interview varied from 59 minutes to one 77 minutes. The highest number of random diagrams assessed in an interview was thirteen. The interviews and focus group were recorded and transcribed verbatim for thematic analysis.

\subsection{Data analysis}

We fully anonymised the transcribed data and imported all into the ATLAS.ti qualitative data analysis software including the participant feedbacks.

We conducted a thematic analysis on the data (Braun and Clarke, 2006), starting with the identification of appropriate quotations in the transcript. For each quotation, as many codes as possible were identified. In the first instance, codes were identified as to whether they represented positive attributes of the diagrammatic representations or negative attributes. It was desired that this first level of coding would be as close as possible to the raw data. As a result, the codes were designed to 
identify whether the related quotation was from Service Users (SU) or Staff. The code contained "Rep", standing for representation stage of the study. This is followed by a code for the specific diagram that the participant was responding to. This varied from "Flct" for flowchart and "DM1" to "DM13" for the random maps used. and the description "Postv" or "Neg" depending on whether the extract from the quotation captures a participant's view of a positive or native attribute of the diagram. Two examples of codes are shown below:

\section{"Staff-Rep-DM2-Postv - I quite like that because that's kind of the language that I would speak and it's good. Reassess, if in doubt go back to assessment."}

"SU-Rep-DM1-Neg - Not a helpful diagram"

This comprehensive approach to coding at this first level was later very helpful during the development of themes. Overall, 377 codes were developed. 276 (73\%) were negative codes including 43 by service users and 101(27\%) positive codes, including 23 by service users. It is important to note that whilst staff data came from face-to-face interviews, the service user data came from a focus group hence the group dynamic with some inherent consensus might explain the fewer codes from the service user data.

The rest of the analysis focused on the search for themes, review of themes and their definition as reported in the section on results.

\section{Results}

The goal of our analysis was to identify what we consider a set of requirements that a diagram or diagramming approach designed for mental health services will have to satisfy to be effective. The thematic analysis employed has led to concepts or themes that were evident from the data, based on the frequency of their occurrence or their relevance to the objective of the analysis and the wider context of the wider research project. It is important to emphasise at this stage that a thematic analysis in the qualitative tradition is not driven by the frequency of occurrence of a theme or concept (Braun and Clarke, 2006; Nowell et al., 2017). Context and relevance are most important.

The results from our analysis are in three parts -1 . Themes that represent what participants liked about the diagrams they were presented with 2 . Themes that represent what participants did not like about the diagrams they were presented with and 3. Merging the likes and dislikes into final set of themes that represent the requirements.

Two main themes represented what participants liked about the diagrams they were presented with Clear logical flow (which included the subthemes "good use of colour", "clear start", "use of pictures", "appropriate level of detail" and "clear name") and Supports decisions about patients (which included one subtheme of being "informative"). Clear logical flow was considered a major theme because not only was it the most frequent (38 out of 101) but it can be seen that many of the other minor themes relate to it. Supports decisions about patients appeared in only four codes, however, our appreciation of the context and observations from the workshops and interviews tell us that it is a major theme.

Four main themes were identified to represent what participants did not like about the diagrams - 1 . Lack clarity of logic (including the subthemes "unhelpful layout", "unhelpful use of colour", "wrong order of activities", "unclear language", "unclear audience", "hard to read", "no feedback loop" and "unclear starting point") 2. Not centred on patients 3. Missing system goals and 4. Missing other parts of system. Lack clarity of logic was the most frequent theme (54 out of 276) but also related to several other codes. Second most frequent was Not centred on patients (45 out of 276). It must also be noted here that there were other themes that have been rejected because they appeared in only one or two codes and the researchers had no strong contextual reasons to include them. In some cases, they may be considered as not too dissimilar from themes that had already been captured. As will be expected, the themes that emerged from what participants did not like only reinforced those themes that describe what they liked.

By combining the two sets of themes, we arrived at four key themes which we propose as key requirements for diagramming in mental health delivery systems - Clear logical flow (with subthemes "good layout", "clear start with feedback", "clear flow of activities", "good use of colour" and "good 
use of language"), Taking account of patient experience, Taking account of wider system and Taking account of system goals. The following subsections will define these themes and provide further details on them.

\subsection{Clear logical flow}

This theme represents the requirement for diagrams of mental health delivery systems to be clear, logical and easy to read. It is an overarching theme that involves several other themes as outlined and illustrated in the subsections below. One of the best illustrations of this theme is provided in the quote below by a staff:
"It's very nice. And so it is telling you without you even having to be told, ...before you even start reading you know you need to act now, no ifs, no buts ..." - Staff-Rep- DM9-Postv

\subsubsection{Start/Feedback}

This theme seemed to have occurred time and again and especially appeared to have caused some frustration to participants. Clearly to be able to easily identify where to start reading a diagram is important to participants as illustrated by this quote:

"I don't know where it starts, I don't know where it's going, I don't know what's going on." - Staff-Rep-DM12-Neg

\subsubsection{Flow}

This theme is about the clear flow of activities through the diagrammatic representation. This was often a source of confusion to some participants. It is an important requirement that a diagram represents the flow of activities in a way that is reasonably consistent with what the reader will expect. That was not the case in this quote:

"It's really interesting as the first port of call is to assess the feeding and provide support to breastfeeding mothers. I would think that the first thing you would want to do, to check for the levels and then provide support later on." - Staff-Rep-DM10-Neg

\subsubsection{Layout}

Good layout is clearly related to the requirement for clear and logical flow in a diagram. The implications of a good layout of a diagram for the ability of reader to comprehend what is communicated is made obvious in the following quote.

"No, they could have just probably have changed the layout to make it better and make it a better read, for flow, for flow wise." - Staf-Rep-DM11-Neg

\subsubsection{Language}

Good use of language is perhaps the most obvious of the requirements. Diagrammatic representations inevitably involve the use of natural language leading to the potential barrier to understanding on the part of the reader. The language used may be context specific, but this requirement reminds the producer of a diagram representation to give careful consideration to the appropriateness of the language involved. The reactions of this staff member may be a helpful reminder.

"Clinical record gets entered into a database as digital ledger. What the hell is a digital ledger?” - Staff-Rep-DM3-Neg

\subsubsection{Colour}

The participants' reaction to colour in diagrams varied from strong dislike to indifference. What was clear and perhaps not surprising was that none of the participants wanted more of it. Some participants had a strict sense of which colours should represent which concepts whilst others did not mind as long 
as it is visually appealing. The quote that follows is an example of a participants who expects that colours should match the concepts they represent.

"I would rather have red at the end rather than purple, purple to me is not more important red ..." - Staff-Rep-DM6-Neg

\subsection{Taking account of patient experience}

The theme of patient experience was the least represented in all the diagrams used for elicitation and appeared the most important to participants. The theme was highly frequent in both the staff and service user data. It is often raised in the sense that patients are the very reason why the service exists hence consideration of processes and all aspects of the system needs to be cognisant of patients and by implication their families as well.

"I would say the patient. Where's the service user? $i$ think the diagrammatic representation should have the patient at the centre." - Staff-Rep-Flct-Neg

\subsection{Taking account of wider system}

This theme developed predominantly out of staff data. Perhaps this is because it is the staff that bear the responsibility for ensuring that service users are appropriately discharged from in-patient wards. Some staff have referred to this by recalling difficulties they often encounter at the point of discharge because community services are not sometimes ready to provide the support needed when a service user is discharged.

"I am guessing there are other parts of the system. That's looking at staff but you could almost look at the buildings as well and say the same thing." - Staff-Rep-Flct-Neg

\subsection{Highlighting system goals}

This theme may relate to that of "taking account of the patient's experience". This is because in one sense, the goal of the system is to care for patients. There is, however, a sense in which the goal of the system includes but not limited to caring for patients. There may be other goals such as financial, staffing or policy goals which may need to be balanced with patient care for the long-term sustainability of the system. This quote from a staff, however, may be referring to patient related goal.

\section{"My key thing is I want to know what the purpose of the admission is in the flowchart" - Staff-Rep-Flct-Neg}

One of the reasons that this has been considered a major theme is because goals in general, were one of the key components of the delivery system identified in the first stage of the wider research project (Komashie et al., 2017).

It is not difficult to imagine that if a diagram is produced or a diagramming approach for mental health services is developed to meet these requirements, it may go a long way to help in communicating the complexities of the delivery system to a wide range of stakeholders and better support service redesign and improvement.

\section{Discussions}

\subsection{Requirements for diagramming in mental health}

The results presented above clearly show that service users and staff (clinicians and managers) in mental health do have considerably strong views on what constitutes a good diagrammatic representation or at least what will make them interested in reading a diagrammatic representation.

The results show four main themes which were refer to as the requirements for diagramming - Clear logical flow, Taking account of patient experience, Taking account of wider system and Highlighting system goals - and five lower level requirements - Good layout, Good use of language, Clear start with feedback, Clear flow of activities and Good use of colour. 
We believe that this set of requirements can raise a useful set of questions for anyone who needs to produce any kind of diagram for the purpose of communicating an aspect of the delivery system.

\subsection{Findings in context}

The current findings form a logical progression on previous work relating to mental health system design reported in the design literature by the same authors. The authors studied mental health delivery systems to understand the key components that interact within them (Komashie et al., 2017). Through stories that service users and clinicians told in focus groups and interviews, the authors identified ten system components - Patients, Conditions, Goals, Resources, Processes, Interventions, Family/Friends, Information and External agencies (or system environment). They continued to study the nature of the relationships between these components (Komashie and Clarkson, 2018) as a step towards the development of a diagrammatic approach to modelling mental health delivery systems. This further establishes the relevance of the present results which together points to the next steps of defining and developing a diagrammatic approach for mental health delivery systems.

Jun and colleagues conducted a similar study into understanding how healthcare staff perceived the usefulness and ease of use of various types of standard diagramming types including flowcharts, swimlane diagrams, communication diagrams and state transition diagrams (Jun et al., 2009). They found that of the eight different diagram types presented to participants, flowcharts were the most familiar and came up as favourite both in terms of usability and perceived utility. The current results add to Jun et al., by broadening the representation to include service users and taking a wider view of diagrams beyond standard diagram types. Our results should therefore be useful to those wanting to apply standard diagram types or develop a new way of diagramming in mental health.

Similarly, extensive research by Allan Blackwell has focussed on the cognitive dimensions of notations and has covered a wide range of the use and development of diagrams. A recent work on pattern language for diagrams presented a set of requirements for developing a diagrammatic representational system (Blackwell, 2019). Blackwell found that users of diagrams undertake three core activities - build information structures, read information structures and share information structures. Whilst they undertake these activities, users have experiences that include vision, interaction, meaning, thinking, process and creativity. He argues that the development of an effective diagrammatic representational system must take these activities and experiences into account. Though Blackwell's work was not focused on mental health services, we find some complementarity between his work and our present findings. For instance, though Blackwell identifies building of information structures as one of the activities users undertake with diagrams, it does not define how that must be done. Our requirement of clear logical flow and its sub-requirements provide a more detailed guideline for constructing those information structures and if well undertaken, should facilitate the information reading activity that Blackwell identifies.

\subsection{Implications}

The identification of requirements for diagramming in the design of mental health delivery services as reported in this paper may have implications for a number of stakeholders. First, this could become a guide to those involved in developing diagrammatic description of services. This may include service re-design teams in NHS hospitals, improvement practitioners and teams in the Central Commissioning facilities (CCG) in the NHS who support service re-design.

Secondly, this may become an assessment tool for diagrams developed in mental health and possibly healthcare services in general. In this regard, we propose a matrix style framework (Figure 3 ) which could help judge the quality and relevance of a diagrammatic representation. Matrix 1 in Figure 3 represents a diagrammatic model that effectively provides clear logical flow but remains lacking in all the remaining requirements. Such a diagram may be considered to have moderate quality and little relevance to a mental health context. A representation with clear logical flow that takes account of wider system may be considered to have high diagrammatic quality but still little relevance (matrix 2). Taking account of system goals in addition to matrix 2 will mean the representation has high diagrammatic quality and moderate relevance to the mental health context 
(matrix 3). Finally, matrix 4 will refer to the ideal representation of high diagrammatic quality and highly relevant to the mental health context.

We acknowledge that this is a proposal and will require further testing to establish any validity.
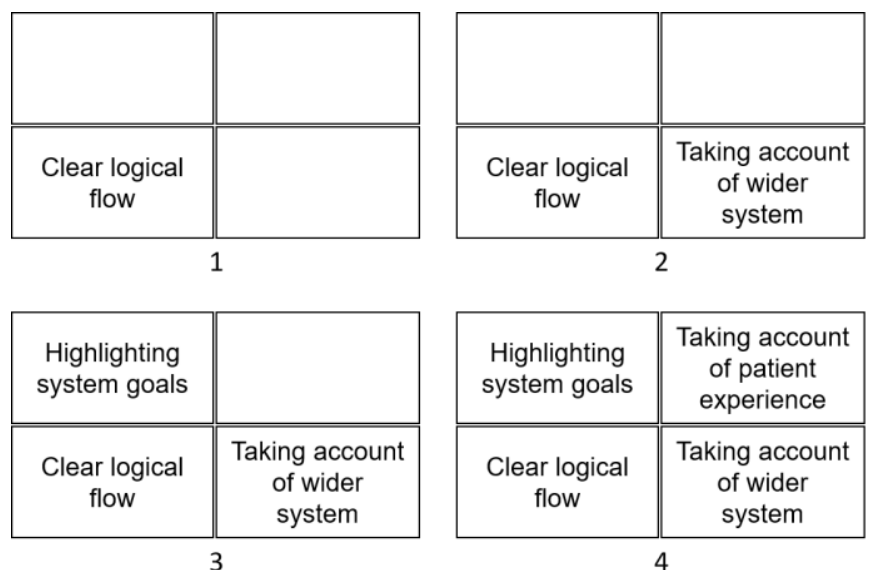

Figure 3. A diagrammatic quality and relevance matrix: matrix 1 represents good quality diagram with little relevance to mental health, matrix 2 represents high quality diagram with little relevance to mental health, matrix 3 represents high quality diagram with moderate relevance, matrix 4 represents high quality diagram, highly relevant to mental health

\subsection{Limitations}

It is important to note that this study, like most research studies has several limitations. In the first place, our sampling process may be most appropriately described as convenience sampling. This has considerable limitations in terms of the potential to bias the outcome. Convenience samples are known to badly represent the population of interest and prone to the inclusion of outlines due to self-selection (Etikan et al., 2016).

Secondly, we only included two groups of stakeholders. Whilst we believe that service users and staff form major stakeholders, it is possible that including other stakeholders like family member, funders, and policy makers or even the police with different perspectives could have changed the outcome significantly. The sample size of six service users in a focus group and five staff interviews for the work reported in this paper represents a further limitation.

\section{Conclusions}

We report the outcome of a qualitative thematic analysis of service user and staff interviews. We identified four key themes that we consider to form a set of requirements for diagramming in mental health services. We have suggested that these requirements may be a helpful framework for assessing the quality and relevance of diagrammatic representations in mental health. These findings provide a necessary basis for beginning to formulate a diagrammatic approach for mental service design, which forms an obvious future research opportunity in which we are engaged.

\section{Acknowledgements}

This Research was supported by the National Institute for Health Research (NIHR) Collaboration for Leadership in Applied Health Research and Care (CLAHRC) for the East of England at the Cambridgeshire and Peterborough NHS Foundation Trust. The views expressed are those of the authors and not necessarily those of the NHS, the NIHR or the Department of Health. The authors will also like to acknowledge the contributions of the Service User Advisory Group (SUAG) for their valuable contributions in shaping the DIAGRAMS research.

\section{References}

del Aguila, I.M., Palma, J. and Tunez, S. (2014), "Milestones in Software Engineering and Knowledge Engineering History: A Comparative Review", The Scientific World Journal, Vol. 2014, pp. 1-10. 
Blackwell, A. (2019), “A Pattern Language for the Design of Diagrams”, In: Richards, C. (Ed.), Elements of Diagramming: Design Theories, Analyses and Methods, Routledge, p. 250.

Braun, V. and Clarke, V. (2006), "Using thematic analysis in psychology", Qualitative Research in Psychology, Vol. 3 No. 2, pp. 77-101.

Clarkson, P.J. (2018), "What has engineering design to say about healthcare improvement?", Design Science, Vol. 4, pp. 1-35.

Clarkson, P.J. et al. (2017), Engineering Better Care: A Systems Approach to Health and Care Design and Continuous Improvement, Royal Academy of Engineering, London,

Colligan, L. et al. (2010), "Does the process map influence the outcome of quality improvement work? A comparison of a sequential flow diagram and a hierarchical task analysis diagram", BMC Health Services Research, Vol. 10 No. 1, p. 7.

Crilly, N., Blackwell, A.F. and Clarkson, P.J. (2006), "Graphic elicitation: using research diagrams as interview stimuli”, Qualitative Research, Vol. 6, pp. 1-27.

Department of Health and Design Council (2003), Design for Patient Safety: A System-Wide Design-Led Approach to Tackling Patient Safety in the NHS, London, UK.

Etikan, I., Musa, S.A. and Alkassim, R.S. (2016), "Comparison of Convenience Sampling and Purposive Sampling", American Journal of Theoretical and Applied Statistics, Vol. 5 No. 1, p. 1.

Graham, B. (2008), “The Roots of the Business Process Mapping”, BP Trends, No. June, pp. 1-6.

Jun, G.T. et al. (2009), "Health care process modelling: which method when?", International Journal for Quality in Health Care, Vol. 21 No. 3, pp. 214-224.

Komashie, A. and Clarkson, P.J. (2018), "Designing mental health delivery systems: Describing the relationship between system components", Proceedings of the 15th International Design Conference, DESIGN 2018, Vol. 6, pp. 2669-2680.

Komashie, A. et al. (2017), "Designing mental health delivery systems: Where do we start?", DS 87-3 Proceedings of the 21st International Conference on Engineering Design (ICED 17) Vol 3: Product, Services and Systems Design, Vancouver, Canada, 21-25.08.2017, Vol. 3.

Mincarone, P. et al. (2018), "Standardized languages and notations for graphical modelling of patient care processes: A systematic review", International Journal for Quality in Health Care, Vol. 30 No. 3, pp. 169-177.

Nowell, L.S. et al. (2017), "Thematic Analysis: Striving to Meet the Trustworthiness Criteria", International Journal of Qualitative Methods, Vol. 16 No. 1, pp. 1-13.

Object Management Group (2011), "OMG Systems Modelling Language (OMG SysML) Version 1.3”, USA.

Purcell, A.T. and Gero, J.S. (1998), "Drawings and the design process”, Design Studies, Vol. 19 No. 4, pp. 389-430.

Ram, S. and Liu, J. (2008), “A Semiotics Framework for Analyzing Data Provenance Research", Journal of Computing Science and Engineering, Vol. 2 No. 3, pp. 221-248.

Reid, P.P. et al. (2005), Building a Better Delivery System: A New Engineering/Health Care Partnership, The National Academy Press, Washington D.C.

Staniszewska, S. et al. (2011), "Developing the evidence base of patient and public involvement in health and social care research: The case for measuring impact", International Journal of Consumer Studies, Vol. 35 No. 6, pp. 628-632.

Störrle, H. et al. (2018), "Poster: How do modelers read UML diagrams?: Preliminary results from an eyeTracking study", Proceedings - International Conference on Software Engineering, pp. 396-397.

Störrle, H. and Fish, A. (2013), "Towards an operationalization of the 'Physics of Notations' for the analysis of visual languages", Lecture Notes in Computer Science (Including Subseries Lecture Notes in Artificial Intelligence and Lecture Notes in Bioinformatics), Vol. 8107 LNCS, pp. 104-120.

Tversky, B. (2011), "Visualizing thought", Topics in Cognitive Science, Vol. 3, pp. 499-535.

Tversky, B. (2015), "The cognitive design of tools of thought", Review of Philosophy and Psychology, Vol. 6 No. 1, pp. 99-116.

WHO (2000), Health Systems: Improving Performance, World Health Report 2000, WHO, Geneva, Switzerland.

Yusuf, S., Kagdi, H. and Maletic, J.I. (2007), “Assessing the comprehension of UML class diagrams via eye tracking”, IEEE International Conference on Program Comprehension, pp. 113-122. 